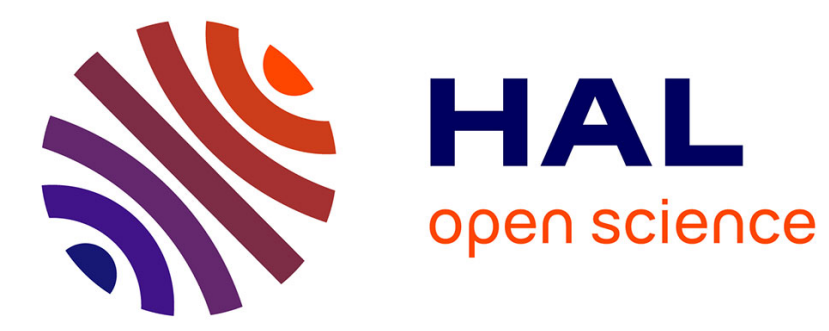

\title{
Sur les processus arithmétiques liés aux diviseurs
}

Régis de La Bretèche, Gérald Tenenbaum

\section{To cite this version:}

Régis de La Bretèche, Gérald Tenenbaum. Sur les processus arithmétiques liés aux diviseurs. Advances in Applied Probability, 2016, 48 (A), pp.63-76. 10.1017/apr.2016.42 . hal-01281742

\section{HAL Id: hal-01281742 \\ https://hal.science/hal-01281742}

Submitted on 2 Mar 2016

HAL is a multi-disciplinary open access archive for the deposit and dissemination of scientific research documents, whether they are published or not. The documents may come from teaching and research institutions in France or abroad, or from public or private research centers.
L'archive ouverte pluridisciplinaire HAL, est destinée au dépôt et à la diffusion de documents scientifiques de niveau recherche, publiés ou non, émanant des établissements d'enseignement et de recherche français ou étrangers, des laboratoires publics ou privés. 


\title{
Sur les processus arithmétiques liés aux diviseurs
}

\author{
R. de la Bretèche \& G. Tenenbaum
}

To Nick Bingham, with the belief that friendship is a non-random process

\begin{abstract}
For natural integer $n$, let $D_{n}$ denote the random variable taking the values $\log d$ for $d$ dividing $n$ with uniform probability $1 / \tau(n)$. Then $t \mapsto \mathbb{P}\left(D_{n} \leqslant n^{t}\right)(0 \leqslant t \leqslant 1)$ is an arithmetic process with respect to the uniform probability over the first $N$ integers. It is known from previous works that this process converges to a limit law and that the same holds for various extensions. We investigate the generalized moments of arbitrary orders for the limit laws. We also evaluate the mean value of he two-dimensional distribution function $\mathbb{P}\left(D_{n} \leqslant n^{u}, D_{\left\{n / D_{n}\right\}} \leqslant n^{v}\right)$.
\end{abstract}

Key-words. Arc-sine law; arithmetic model; arithmetic process; Diriclet laws; divisors.

2010 Mathematics Subject Classification. Primary 11K65; Secondary 11N37; 11N64.

\section{Introduction et énoncés des résultats}

Soit $E \subset[0,1]^{\mathbb{R}}$ un espace de fonctions métrisable. Une suite $\{t \mapsto f(n, t)\}_{n=1}^{\infty}$ de fonctions de $E$ peut être considérée comme un processus arithmétique à valeurs dans $E$ relativement à la mesure de comptage $\nu_{N}$ uniforme sur $\Omega_{N}:=\left\{n \in \mathbb{N}^{*}: 1 \leqslant n \leqslant N\right\}$. Notant $\mathcal{B}(E)$ la tribu des boréliens de $E$ et posant

$$
\mu_{N}(A):=\nu_{N}\{n: f(n, \cdot) \in A\} \quad(A \in \mathcal{B}(E))
$$

on dit que $f(n, t)$ converge en loi vers un processus $\mu$ à valeurs dans $E$ si l'on a $\lim _{N \rightarrow \infty} \mu_{N}(A)=\mu(A)$ pour tout borélien $A$ de $\mathcal{B}(E)$ tel que $\mu(\partial A)=0$. On écrit alors

$$
\mu_{N} \Rightarrow \mu \quad(N \rightarrow \infty) .
$$

On étend trivialement ces définitions au cas d'une suite double $\left\{t \mapsto f_{N}(n, t)\right\}$.

Si $(\Omega, \mathcal{A}, \mathbb{P})$ est un espace probabilisé, $X: t \mapsto X_{t}$ est un processus sur $\Omega$ à valeurs dans $E$, et si $\mu_{N}=\nu_{N} \circ f_{N}^{-1} \Rightarrow \mathbb{P} \circ X^{-1}$, on dit aussi que que $f_{N}$ constitue un modèle arithmétique de $X$.

La modélisation de processus classiques par des fonctions arithmétiques additives a été abondamment étudiée dans la littérature - voir par exemple [5], [6], [16], [14], [15], [20], [12], et les références incluses dans ces travaux. Considérons une suite $\left\{h_{N}\right\}_{N \geqslant 1}$ de fonctions fortement additives et posons

$$
\begin{aligned}
& B_{N}(z):=\sum_{p \leqslant z} \frac{h_{N}(p)}{p}, \quad B_{N}:=B_{N}(N), \\
& x_{N}(t):=\sup \left\{z: B_{N}(z)^{2} \leqslant t B_{N}^{2}\right\} \quad(0 \leqslant t \leqslant 1), \\
& W_{N}(n, t):=\frac{1}{B_{N}}\left(\sum_{p \leqslant x_{N}(t), p \mid n} h(p)-\sum_{p \leqslant x_{N}(t)} \frac{h_{N}(p)}{p}\right) .
\end{aligned}
$$

Ici et dans la suite, la lettre $p$ est réservée pour désigner un nombre premier. Précisant des résultats de Kubilius [15] et Billingsley [5], Philipp [20] a montré en 1973 que $W_{N}$ 
constitue un modèle arithmétique du processus de Wiener $W$ dans $E=\mathcal{C}[0,1]$ muni de la topologie de la convergence uniforme dès que que la condition de Lindeberg

$$
(\forall \varepsilon>0) \quad \sum_{\substack{p \leqslant N \\ h_{N}(p) \mid>\varepsilon B_{N}}} \frac{h_{N}(p)^{2}}{p}=o\left(B_{N}^{2}\right) \quad(N \rightarrow \infty)
$$

est satisfaite. Une démonstration alternative a été produite par Babu [1]. Timofeev \& Usmanov [24] ont prouvé en 1982 que la condition (1·1) est aussi nécessaire.

Soient $\tau(n, z):=\sum_{d \mid n, d \leqslant z} 1, \tau(n):=\tau(n, n)(n \geqslant 1, z \geqslant 1)$. Manstavičius [17] a établi la convergence $U_{N} \Rightarrow W$, où ${ }^{(1)}$

$$
U_{N}(n, t):=\frac{\log \tau\left(n,(\log N)^{t}\right)-t(\log 2) \log _{2} N}{(\log 2) \sqrt{\log _{2} N}}
$$

est considéré comme un processus à valeurs dans l'espace de Skorokhod $\mathbf{D}=\mathbf{D}[0,1]$, constitué de l'ensemble des fonctions continues à droite et possédant des limites à gauche, muni de la topologie éponyme. Les résultats de ce type sont obtenus en exploitant l'approximation naturelle de $\tau(n, z)$ par $2^{\omega(n, z)}$, où $\omega(n, z):=\sum_{p \mid n, p \leqslant z} 1$.

Des phénomènes plus exotiques apparaissent en analysant le processus

$$
X_{n}(t):=\frac{\tau\left(n, n^{t}\right)}{\tau(n)} \quad(0 \leqslant t \leqslant 1),
$$

dont Dress, Deshouillers \& Tenenbaum [7] ont montré que l'espérance des lois marginales d'ordre 1 tend vers la fonction de répartition de la loi de l'arcsinus : on a en fait, plus précisément,

$$
\mathbb{E}_{N}\left(X_{n}(t)\right)=\frac{2}{\pi} \arcsin \sqrt{t}+O\left(\frac{1}{\sqrt{\log N}}\right) \quad(0 \leqslant t \leqslant 1, N \geqslant 2),
$$

où $\mathbb{E}_{N}$ désigne l'espérance relativement à la mesure $\nu_{N}$. Il serait incidemment intéressant de proposer une explication probabiliste pour l'apparition de cette loi dans un tel contexte arithmétique : peut-on, par exemple, modéliser le membre de gauche de (1.2) par une loi de fluctuations dans un jeu de pile ou face comme dans [9] (chap. III) ou, plus généralement, par celle du nombre des sommes partielles positives d'une suite de variables aléatoires indépendantes comme dans [8] ? Voir également [13].

Plus généralement, on peut considérer le processus

$$
X_{n}(g, t):=\frac{\sum_{d \mid n, d \leqslant n^{t}} g(d)}{\sum_{d \mid n} g(d)}
$$

où $g$ est une fonction multiplicative positive ou nulle. Dans ce cadre, la convergence en loi a été établie par Manstavičius \& Timofeev [18] lorsque $g(p)=\kappa>0$ est indépendant de $p$. Posant $\mu_{N}:=\nu_{N} \circ X_{n}^{-1}$ et $\mu:=\lim _{N} \mu_{N}$, il résulte alors d'une estimation effective de Bareikis \& Manstavičius [4] que, notant $\sigma:=1 /(1+\kappa)$,

$$
\int_{\mathbf{D}} \varphi(t) \mathrm{d} \mu(\varphi)=\frac{\sin (\pi \sigma)}{\pi} \int_{0}^{t} \frac{\mathrm{d} v}{v^{\sigma}(1-v)^{1-\sigma}}
$$

généralisant ainsi la formule $(1 \cdot 2)$, relative au cas $\kappa=1$.

1. Dans tout ce travail, nous notons $\log _{k}$ la $k$-ième itérée de la fonction logarithme. 
En fait, le résultat de [4] fournit une estimation de l'espérance de $X_{n}(g, t)$ sous l'hypothèse plus faible que $g(p)$ est assez proche de $\kappa$, en un sens en moyenne précisé dans le travail. Une estimation de la vitesse de convergence est également fournie.

Dans le cas $g=\mathbf{1}$, une autre spécificité de la mesure limite $\mu$ est que, pour tout couple $(s, t) \in] 0,1\left[2\right.$, la suite des accroissements $X_{n}(t)-X_{n}(s)$ converge en loi vers une variable aléatoire dont les points de croissance appartiennent à l'ensemble $\mathcal{R}$ des rationnels dyadiques, autrement dit

$$
z \mapsto \mu\{\varphi \in \mathbf{D}: \varphi(t)-\varphi(s) \leqslant z\}
$$

est une mesure atomique à support dans $\mathcal{R}$. Cela découle directement des résultats du second auteur dans [21] et implique en particulier que $\mu \neq W$. On déduit également des estimations de [21] que

$$
(\forall t \in] 0,1[) \quad \varphi^{\prime}(t)=0 \quad(\mu-\mathrm{pp}),
$$

et l'on peut établir que les relations $\varphi^{\prime}(0+)=-\infty, \varphi^{\prime}(1-)=+\infty$ ont lieu $\mu$-presque sûrement.

Dans [22], Tenenbaum a montré que les conditions suivantes, concernant la fonction multiplicative positive ou nulle $g$, sont suffisantes pour impliquer la convergence en loi de $X_{n}(g, t)$ :

$$
\left\{\begin{array}{l}
\forall h, k(0 \leqslant h \leqslant k), \forall \alpha \in] 0,1\left[\exists \lambda(\alpha ; h, k): \lim _{N \rightarrow \infty} \sum_{N^{\alpha}<p \leqslant N} \frac{g(p)^{h}}{p\{1+g(p)\}^{k}}=\lambda(\alpha ; h, k),\right. \\
\lim _{\alpha \rightarrow 0+} \lambda(\alpha ; 1,2)=\infty
\end{array}\right.
$$

De plus, sous ces hypothèses, le support de la loi limite $\mu$ est inclus dans l'ensemble $\mathcal{C}[0,1]$ des fonctions continues sur $[0,1]$.

Ainsi, par exemple, une fonction $g$ telle que $g(p)=\frac{1}{2}\left\{1+(-1)^{(p-1) / 2}\right\}$ entre dans le champ d'application; comme on vérifie aisément que les hypothèses de [4] sont satisfaites dans ce cas, il s'ensuit que la relation (1.4) est valide avec $\kappa=\frac{1}{2}$, et donc $\sigma=\frac{2}{3}$.

Dans leur récents articles [2] et [3], Bareikis et Mačiulis ont évalué le second moment de la loi limite $\mu$ sous l'hypothèse de régularité en moyenne de $g(p)$. Un premier objectif du présent travail consiste à évaluer, pour $g=\mathbf{1}$ - voir cependant la remarque (iii) infra -, les moments généralisés d'ordre $r \geqslant 1$ quelconque, soit, pour tout $r$-uplet $\left.\boldsymbol{t}=\left(t_{0}, \ldots, t_{r-1}\right) \in\right] 0,1\left[^{r}\right.$

$$
S_{r}(N ; \boldsymbol{t}):=\mathbb{E}_{N}\left(\prod_{0 \leqslant j<r} X_{n}\left(t_{j}\right)\right)=\frac{1}{N} \sum_{n \leqslant N} \prod_{0 \leqslant j<r} \frac{\tau\left(n, n^{t_{j}}\right)}{\tau(n)} .
$$

Pour énoncer notre résultat, nous introduisons le développement en base 2 d'un entier $h$, soit

$$
h=\sum_{j \geqslant 0} \varepsilon_{j}(h) 2^{j},
$$

où $\varepsilon_{j}(h) \in\{0,1\}$. Nous dirons qu'un entier $h$ est dominé par un entier $\ell$ et nous écrirons $h \prec \ell$ si $\varepsilon_{j}(h) \leqslant \varepsilon_{j}(\ell)$ pour tout $j$. Nous posons encore $R:=2^{r}-1$. Pour tout vecteur $\boldsymbol{v}=\left(v_{1}, \ldots, v_{R}\right) \in \mathbb{R}^{R}$, nous notons $\mathfrak{s}(\boldsymbol{v})$ la somme, et $\wp(\boldsymbol{v})$ le produit, des coordonnées de $\boldsymbol{v}$. Nous établissons l'estimation suivante.

Théorème 1.1. Nous avons, uniformément pour $N \geqslant 2$ et $\boldsymbol{t} \in[0,1]^{r}$,

$$
S_{r}(N ; \boldsymbol{t})=\frac{1}{\Gamma\left(1 / 2^{r}\right)^{2^{r}}} \int_{D(\boldsymbol{t})} \frac{\mathrm{d} \boldsymbol{v}}{\wp(\boldsymbol{v})^{1-1 / 2^{r}}\{1-\mathfrak{s}(\boldsymbol{v})\}^{1-1 / 2^{r}}}+O\left(\frac{1}{(\log N)^{1 / 2^{r}}}\right)
$$

où l'on a posé $\mathrm{d} \boldsymbol{v}=\mathrm{d} v_{1} \cdots \mathrm{d} v_{R}$ et

$$
D(\boldsymbol{t}):=\left\{\boldsymbol{v}=\left(v_{1}, \ldots, v_{R}\right) \in[0,1]^{R}: \sum_{1 \leqslant h \leqslant R} \varepsilon_{j}(h) v_{h} \leqslant t_{j}(0 \leqslant j<r), \mathfrak{s}(\boldsymbol{v}) \leqslant 1\right\} .
$$


Remarques. (i) Pour $r=1$ nous retrouvons bien (1·2).

(ii) Pour $r=2$ et $t_{0}=t_{1}$, notre résultat coïncide avec celui de [2] (th.2.1). En effet, lorsque $t=t_{0}=t_{1} \leqslant \frac{1}{2}$, l'égalité des termes principaux est obtenue en insérant dans le domaine d'intégration de (1.5) la condition de symétrie $v_{2} \leqslant v_{1}$. On vérifie aisément que, lorsque $t>\frac{1}{2}$, le terme principal indiqué dans [2] est identique au nôtre.

(iii) Notre méthode peut être généralisée sans difficulté majeure à des hypothèses en moyenne du type de celles de Bareikis \& Manstavičius dans [4]. Avec la définition (1.3) et lorsque la fonction multiplicative positive ou nulle $g$ est telle que $g(p)$ est assez proche en moyenne d'une constante $\kappa>0$, nous obtenons alors

$$
\mathbb{E}_{N}\left(\prod_{0 \leqslant j<r} X_{n}\left(g, t_{j}\right)\right)=J(\kappa ; \boldsymbol{t})+O\left(\frac{1}{(\log N)^{\sigma^{r} \min \left(1, \kappa^{r}\right)}}\right)
$$

où l'on a posé $\sigma:=1 /(1+\kappa)$,

$$
J(\kappa ; \boldsymbol{t}):=\frac{1}{\prod_{0 \leqslant h \leqslant R} \Gamma\left(\kappa^{s(h)} \sigma^{r}\right)} \int_{D(\boldsymbol{t})} \frac{\mathrm{d} \boldsymbol{v}}{\prod_{1 \leqslant h \leqslant R} v_{h}^{1-\kappa^{s(h)} \sigma^{r}}\{1-\mathfrak{s}(\boldsymbol{v})\}^{1-\sigma^{r}}},
$$

la notation $s(h):=\sum_{j \geqslant 0} \varepsilon_{j}(h)(1 \leqslant h \leqslant R)$ désignant la somme des chiffres de l'entier $h$ dans le développement en base 2 . Pour $r=2, t_{0}=t_{1}$, cette estimation coïncide exactement avec celle de Bareikis et Mačiulis dans [3]. Nous n'explicitons pas les détails de la preuve de (1.6) dans le présent travail.

La moyenne $S_{r}(N ; \boldsymbol{t})$ peut donc être interprétée comme l'espérance, relativement à la mesure de comptage $\nu_{N}$, de la probabilité

$$
\mathbb{P}\left(D_{n, 0} \leqslant n^{t_{0}}, \ldots, D_{n, r-1} \leqslant n^{t_{r-1}}\right)
$$

lorsque les variables aléatoires $D_{n, j}$ sont équiréparties sur les diviseurs de $n$ et indépendantes. Notre second résultat consiste à explorer une situation de dépendance. Nous nous restreignons au cas $r=2$, mais notre méthode fonctionne sans changement majeur dans le cas général.

Des hypothèses de cette nature ont été considérées par Nyandwi \& Smati dans [19], où le modèle choisi est celui de la probabilité uniforme sur l'ensemble des couples $\left(d, d^{\prime}\right)$ tels que $d d^{\prime} \mid n$. Un tel dispositif est incompatible avec l'équirépartition pour la loi marginale du premier diviseur. Un modèle plus naturel de dépendance consiste à choisir un premier diviseur $D_{n}$ de $n$ avec équiprobabilité, puis un diviseur $\Delta_{n}$ de $n / D_{n}$ selon le même mode. La loi conjointe de $\left(D_{n}, \Delta_{n}\right)$ est alors donnée par

$$
\mathbb{P}\left(D_{n}=d, \Delta_{n}=m\right)= \begin{cases}\frac{1}{\tau(n) \tau(n / d)} & \text { si } m \mid n / d, \\ 0 & \text { dans le cas contraire. }\end{cases}
$$

Il s'agit donc d'un tirage de deux diviseurs consécutifs, sans remise. Contrairement à la situation considérée dans [19], la loi de $D_{n}$ demeure ici uniforme.

La valeur moyenne de la fonction de répartition bidimensionnelle est donnée par la quantité

$$
S(N ; u, v)=\frac{1}{N} \sum_{n \leqslant N} \mathbb{P}\left(D_{n} \leqslant n^{u}, \Delta_{n} \leqslant n^{v}\right) .
$$

Nous obtenons le résultat suivant. 
Théorème 1.2. Nous avons, uniformément pour $0 \leqslant u, v \leqslant 1$

$$
S(N ; u, v)=\frac{1}{\Gamma\left(\frac{1}{4}\right)^{2} \Gamma\left(\frac{1}{2}\right)} \int_{D_{u, v}} \frac{\mathrm{d} s \mathrm{~d} t}{s^{1 / 2} t^{3 / 4}(1-s-t)^{3 / 4}}+O\left(\frac{1}{(\log N)^{1 / 4}}\right),
$$

où l'on a posé

$$
D_{u, v}:=\{(s, t) \in[0, u] \times[0, v]: s+t \leqslant 1\} .
$$

Nous avons

$$
\mathbb{P}\left(\Delta_{n}=m\right)=\frac{1}{\tau(n)} \sum_{s \mid n / m} \frac{1}{\tau(s m)}=\frac{1}{\tau(n)} \prod_{\substack{p^{\nu}\left\|n \\ p^{\mu}\right\| m}} \sum_{\nu-\mu \leqslant j \leqslant \nu} \frac{1}{j+1} \quad(m \mid n) .
$$

On vérifie que

$$
\begin{aligned}
\sum_{m \mid n} \prod_{\substack{p^{\nu}\left\|n \\
p^{\mu}\right\| m}} \sum_{\nu-\mu \leqslant j \leqslant \nu} \frac{1}{j+1} & =\prod_{p^{\nu} \| n}\left(\sum_{0 \leqslant \mu \leqslant \nu} \sum_{\nu-\mu \leqslant j \leqslant \nu} \frac{1}{j+1}\right) \\
& =\prod_{p^{\nu} \| n} \sum_{0 \leqslant j \leqslant \nu} \frac{1}{j+1} \sum_{\nu-j \leqslant \mu \leqslant \nu} 1=\tau(n) .
\end{aligned}
$$

Il est à noter que la loi de $\Delta_{n}$ n'est pas symétrique par rapport à $\sqrt{n}$.

Nous déduisons immédiatement du Théorème 1.2 une estimation de la valeur moyenne de la loi du second diviseur $\Delta_{n}$. Nous posons

$$
S(N ; v):=S(N ; 1, v)=\frac{1}{N} \sum_{n \leqslant N} \mathbb{P}\left(\Delta_{n} \leqslant n^{v}\right) .
$$

Corollaire 1.3. La variable aléatoire $\Delta_{n}$ suit en moyenne une loi Bêta de paramètres $\left(\frac{3}{4}, \frac{1}{4}\right)$. Plus précisément, nous avons, uniformément pour $0 \leqslant v \leqslant 1$,

$$
S(N ; v)=\frac{1}{\pi \sqrt{2}} \int_{0}^{v} \frac{\mathrm{d} t}{t^{3 / 4}(1-t)^{1 / 4}}+O\left(\frac{1}{(\log N)^{1 / 4}}\right) .
$$

Remarque. Le terme d'erreur de (1-10) est optimal. Nous avons en effet

$$
S(N ; v)=\frac{1}{N} \sum_{\substack{m d k \leqslant N \\ m \leqslant(d k)^{v /(1-v)}}} \frac{1}{\tau(d k m) \tau(k m)} .
$$

Si $0<v<(\log 2) / \log (2 N)$, seul $m=1$ apparaît dans la sommation. Le membre de droite est donc indépendant de $v$ alors que le terme principal de $(1 \cdot 10)$ est $\sim(2 \sqrt{2} / \pi) v^{1 / 4}$.

L'apparition de lois de Dirichlet dans les deux derniers énoncés induit naturellement la question des liens avec les cadres probabilistes où elles sont classiquement définies, comme par exemple la statistique bayésienne non paramétrique [10] — un challenge supplémentaire pour arithméticiens et probabilistes. 


\section{Démonstration du Théorème 1.1}

La première étape de la démonstration consiste remplacer, dans la somme $S_{r}(N ; \boldsymbol{t})$, les bornes $n^{t_{j}}$ par $N^{t_{j}}$. Notant

$$
S_{r}^{*}(N ; \boldsymbol{t}):=\frac{1}{N} \sum_{n \leqslant N} \prod_{0 \leqslant j<r} \frac{\tau\left(n, N^{t_{j}}\right)}{\tau(n)},
$$

nous avons en effet

$$
S_{r}^{*}(N ; \boldsymbol{t})-S_{r}(N ; \boldsymbol{t}) \leqslant \frac{1}{N} \sum_{0 \leqslant j<r} \sum_{n \leqslant N} \frac{\tau\left(n, N^{t_{j}}\right)-\tau\left(n, n^{t_{j}}\right)}{\tau(n)} \ll \frac{1}{\sqrt{\log N}},
$$

ainsi qu'il a été établi dans la démonstration du théorème de l'arcsinus, correspondant au cas $r=1$ — voir par exemple le chapitre II.6 de [23].

Nous opérons ensuite un paramétrage des diviseurs $d_{j}$ apparaissant dans le développement du produit $\prod_{0 \leqslant j<r} \tau\left(n, N^{t_{j}}\right)$. Nous proposons à cette fin une variante de la définition des ensembles de décomposition unique donnée dans [11].

Soit $r \geqslant 1$. Conservons la notation $R=2^{r}-1$. Nous dirons qu'un $R$-uplet $\boldsymbol{k}=\left(k_{1}, \ldots, k_{R}\right)$ est réduit si l'on a $\left(k_{h}, k_{\ell}\right)=1$ dès que $h \nprec \ell$ et $\ell \nprec h$. À tout $r$-uplet $\boldsymbol{d}=\left(d_{1}, \ldots, d_{r}\right)$, nous pouvons associer bijectivement un $R$-uplet réduit $\boldsymbol{k}$ tel que

$$
\prod_{1 \leqslant h \leqslant R} k_{h}^{\varepsilon_{j}(h)}=d_{j} \quad(0 \leqslant j<r), \quad\left[d_{1}, \cdots, d_{r}\right]=\prod_{1 \leqslant h \leqslant R} k_{h} .
$$

Il s'ensuit que

$$
S_{r}^{*}(N ; \boldsymbol{t})=\frac{1}{N} \sum_{m \geqslant 1} \sum_{k}^{*} \frac{1}{\tau\left(m \prod_{1 \leqslant h \leqslant R} k_{h}\right)^{r}}
$$

où l'astérisque indique que la somme intérieure porte sur les $R$-uplets réduits $\boldsymbol{k} \in\left(\mathbb{N}^{*}\right)^{R}$ satisfaisant les conditions

$$
\left\{\begin{array}{l}
\prod_{1 \leqslant h \leqslant R} k_{h}^{\varepsilon_{j}(h)} \leqslant N^{t_{j}} \quad(0 \leqslant j<r), \\
m \prod_{1 \leqslant h \leqslant R} k_{h} \leqslant N .
\end{array}\right.
$$

Nous pouvons restreindre l'ensemble de sommation aux $R$-uplets $\boldsymbol{k}$ tels que

$$
K:=\prod_{1 \leqslant h \leqslant R} k_{h} \leqslant N / \mathrm{e}
$$

En effet, lorsque cette condition n'est pas remplie, la variable $m$ vaut 1 ou 2 et la contribution correspondante est au plus que l'ordre de grandeur du terme d'erreur annoncé $\mathfrak{R}_{N}:=1 /(\log N)^{1 / 2^{r}}$.

Pour chaque $\delta>0$, posons

$$
\varphi_{\delta}(n):=\prod_{p \mid n}\left(1+\frac{1}{p^{1-\delta}}\right) \quad(n \geqslant 1) .
$$

Il résulte du théorème II.5.2 de [23], de type Selberg-Delange, que l'estimation

$$
\sum_{m \leqslant N / K} \frac{1}{\tau(m K)^{r}}=\frac{H_{0} N}{\Gamma\left(1 / 2^{r}\right) \tau(K)^{r} K\{\log (N / K)\}^{1-1 / 2^{r}}}\left\{g(K)+O\left(\frac{\varphi_{\delta}(K)}{\log (N / K)}\right)\right\}
$$


est valide pour chaque $\delta>0$ fixé et uniformément pour $1 \leqslant K \leqslant N /$ e avec

$$
\begin{aligned}
H_{0} & :=\prod_{p}\left(1-\frac{1}{p}\right)^{1 / 2^{r}} \sum_{\nu \geqslant 0} \frac{1}{(\nu+1)^{r} p^{\nu}}, \\
g(K) & :=\prod_{p^{\kappa} \| K} \frac{\sum_{\nu \geqslant 0}(\kappa+1)^{r} /(\nu+\kappa+1)^{r} p^{\nu}}{\sum_{\nu \geqslant 0} 1 /(\nu+1)^{r} p^{\nu}} .
\end{aligned}
$$

La contribution à $S_{r}^{*}(N ; \boldsymbol{t})$ du terme d'erreur est encore $\ll \mathfrak{R}_{N}$.

En conservant la notation $(2 \cdot 3)$, nous obtenons la contribution principale

$$
\frac{H_{0}}{\Gamma\left(1 / 2^{r}\right)} \sum_{\substack{k_{1} \geqslant 1, \ldots, k_{R} \geqslant 1 \\ K \leqslant N / \mathrm{e}}}^{*} \frac{g(K)}{K \tau(K)^{r}\{\log (N / K)\}^{1-1 / 2^{r}}} .
$$

Notons $\chi\left(k_{1}, \ldots, k_{R}\right)$ la fonction indicatrice des $R$-uplets réduits. Nous évaluons la contribution du terme principal $(2 \cdot 5)$ en sommant successivement sur les variables $k_{R}, \ldots, k_{1}$. L'inversion de l'ordre des indices est justifiée par le fait que $h \prec R$ pour tout $h \in\left[1, R\left[\right.\right.$. On note également que, pour tout $h \in[2, R]$, on a $\chi\left(k_{1}, \ldots, k_{h}\right)=$ $\chi\left(k_{1}, \ldots, k_{h-1}\right) \chi\left(k_{1}, \ldots, k_{h}\right)$. De plus, cette quantité dépend multiplicativement de $k_{h}$ lorsque les autres variables sont fixées.

Posons $K_{h}:=k_{1} \cdots k_{h}(1 \leqslant h \leqslant R)$. Une application immédiate du théorème II.5.2 de [23] (th. II.5.3 dans la seconde édition), de type Selberg-Delange, fournit alors l'existence d'une constante $H_{R}$ et d'une fonction multiplicative $g_{R}$, vérifiant $g_{R}(p)=1+O(1 / p)$, $g_{R}\left(p^{\nu}\right) \ll 1(p \geqslant 2, \nu \geqslant 2)$, telle que l'on ait, pour tout $\delta>0$ fixé,

$$
\begin{aligned}
\sum_{k_{R} \leqslant y} & \frac{g(K) \chi\left(k_{1}, \ldots, k_{R}\right)}{\tau(K)^{r}} \\
& =\frac{H_{R} y \chi\left(k_{1}, \ldots, k_{R-1}\right)}{\Gamma\left(1 / 2^{r}\right) \tau\left(K_{R-1}\right)^{r}(1+\log y)^{1-1 / 2^{r}}}\left\{g_{R}\left(K_{R-1}\right)+O\left(\frac{\varphi_{\delta}\left(K_{R-1}\right)}{1+\log y}\right)\right\}
\end{aligned}
$$

uniformément pour $y \geqslant 1$.

Notant $W_{R-1}:=\left(\log K_{R-1}\right) / \log N$ et $w_{h}:=\left(\log k_{h}\right) / \log N(1 \leqslant h \leqslant R)$, introduisons alors la fonction indicatrice $\vartheta$ de l'ensemble des $R$-uplets $\boldsymbol{w}=\left(w_{0}, \ldots, w_{R-1}\right) \in\left[0,+\infty\left[{ }^{R}\right.\right.$ tels que $\mathfrak{s}(\boldsymbol{w}) \leqslant 1-1 / \log N$ et

$$
\mathfrak{s}_{j}(\boldsymbol{w}):=\sum_{1 \leqslant h \leqslant R} \varepsilon_{j}(h) w_{h} \leqslant t_{j} \quad(0 \leqslant j<r) .
$$

Sous la condition $K_{R-1} \leqslant N / \mathrm{e}$, une sommation d'Abel fournit donc l'estimation uniforme

$$
\begin{aligned}
\sum_{k_{R} \leqslant N / K_{R-1}} \frac{g(K) \chi\left(k_{1}, \ldots, k_{R}\right) \vartheta(\boldsymbol{w})}{\tau(K)^{r}\{\log (N / K)\}^{1-1 / 2^{r}} k_{R}} & \\
= & \frac{H_{R} \chi\left(k_{1}, \ldots, k_{R-1}\right) g_{R}\left(K_{R-1}\right)}{\Gamma\left(1 / 2^{r}\right) \tau\left(K_{R-1}\right)^{r}(\log N)^{1-2 / 2^{r}}} \int_{0}^{1} \frac{\vartheta\left(w_{1}, \ldots, w_{R-1}, v_{R}\right) \mathrm{d} v_{R}}{v_{R}^{1-1 / 2^{r}}\left(1-v_{R}-W_{R-1}\right)^{1-1 / 2^{r}}} \\
& +O\left(\frac{\varphi_{\delta}\left(K_{R-1}\right)}{\tau\left(K_{R-1}\right)^{r}\left\{\log \left(N / K_{R-1}\right)\right\}^{1-1 / 2^{r}}}\right) .
\end{aligned}
$$

On vérifie sans peine que la contribution à l'expression $(2 \cdot 5)$ du terme d'erreur est encore $\ll \mathfrak{R}_{N}$. 
Itérons le procédé en définissant inductivement des fonctions arithmétiques multiplicatives $g_{R-\ell}(1 \leqslant \ell \leqslant R)$. À cette fin, nous écrivons, pour $0 \leqslant \ell \leqslant R-1$,

$$
\sum_{k_{R-\ell} \geqslant 1} \frac{g_{R-\ell}\left(K_{R-\ell}\right) \chi\left(k_{1}, \ldots, k_{R-\ell}\right)}{\tau\left(K_{R-\ell}\right)^{r} k_{R-\ell}^{s}}=\frac{H_{R-\ell}\left(s, K_{R-\ell-1}\right) \chi\left(k_{1}, \ldots, k_{R-\ell-1}\right)}{\tau\left(K_{R-\ell-1}\right)^{r}} \zeta(s)^{1 / 2^{r}}
$$

avec

$$
H_{R-\ell}(s, m):=\prod_{p^{\mu} \| m}\left(1-\frac{1}{p^{s}}\right)^{1 / 2^{r}} \sum_{\nu \geqslant 0} \frac{(\mu+1)^{r} g_{R-\ell}\left(p^{\nu+\mu+1}\right)}{(\nu+\mu+1)^{r} p^{\nu s}} .
$$

Nous définissons alors

$$
H_{R-\ell}:=H_{R-\ell}(1,1), \quad g_{R-\ell-1}(m):=H_{R-\ell}(1, m) / H_{R-\ell}(1,1) .
$$

La fonction $g_{R-\ell}$ est bien multiplicative et vérifie

$$
g_{R-\ell}(p)=1+O(1 / p), \quad g_{R-\ell}\left(p^{\nu}\right) \ll 1 \quad(p \geqslant 2, \nu \geqslant 2) .
$$

Pour $\ell \leqslant R-1$, la $\ell$-ième itération fournit, uniformément pour $y \geqslant 1$,

$$
\begin{aligned}
& \sum_{k_{R-\ell} \leqslant y} \frac{g_{R-\ell}\left(K_{R-\ell}\right) \chi\left(k_{1}, \ldots, k_{R-\ell}\right)}{\tau\left(K_{R-\ell}\right)^{r}} \\
& =\frac{H_{R-\ell} \chi\left(k_{1}, \ldots, k_{R-\ell-1}\right) y}{\Gamma\left(1 / 2^{r}\right) \tau\left(K_{R-\ell-1}\right)^{r}(1+\log y)^{1-1 / 2^{r}}}\left\{g_{R-\ell-1}\left(K_{R-\ell-1}\right)+O\left(\frac{\varphi_{\delta}\left(K_{R-\ell-1}\right)}{1+\log y}\right)\right\},
\end{aligned}
$$

d'où

$$
\begin{aligned}
& \sum_{\substack{k_{R-\ell} \geqslant 1, \cdots, k_{R} \geqslant 1 \\
K \leqslant N / \mathrm{e}}} \frac{g(K) \chi\left(k_{1}, \ldots, k_{R}\right) \vartheta(\boldsymbol{w}) K_{R-\ell-1}}{\tau(K)^{r}\{\log (N / K)\}^{1-1 / 2^{r} K}} \\
&=\frac{H_{R} \cdots H_{R-\ell} \chi\left(k_{1}, \ldots, k_{R-\ell-1}\right) g_{R-\ell}\left(K_{R-\ell-1}\right)}{\Gamma\left(1 / 2^{r}\right)^{\ell} \tau\left(K_{R-\ell-1}\right)^{r}(\log N)^{1-(\ell+2) / 2^{r}}} \\
& \int_{[0,1]^{\ell+1}} \frac{\vartheta\left(w_{1}, \ldots, w_{R-\ell-1}, v_{R-\ell}, \ldots, v_{R}\right) \mathrm{d} v_{R-\ell} \cdots \mathrm{d} v_{R}}{\left(v_{R-\ell} \cdots v_{R}\right)^{1-1 / 2^{r}}\left(1-v_{R-\ell}-\ldots-v_{R}-W_{R-\ell-1}\right)^{1-1 / 2^{r}}} \\
&+O\left(\frac{\varphi_{\delta}\left(K_{R-\ell-1}\right)}{\tau\left(K_{R-\ell-1}\right)^{r}\left\{\log \left(N / K_{R-\ell-1}\right)\right\}^{1-(\ell+1) / 2^{r}}}\right) .
\end{aligned}
$$

La sommation d'Abel est justifiée par le fait que, lorsque toutes les autres variables sont fixées, on a $\vartheta(\boldsymbol{w})=\mathbf{1}_{[0, Z]}\left(w_{R-\ell}\right)$ où $Z$ est une fonction affine par morceaux de $w_{1}, \ldots, w_{R-\ell-1}, w_{R-\ell+1}, w_{R}$.

La contribution à l'expression $(2 \cdot 5)$ du terme d'erreur est encore $\ll \mathfrak{R}_{N}$. L'intégrale apparaissant dans le terme principal est

$$
\ll\left(\frac{\log N}{\log \left(N / K_{R-\ell-1}\right)}\right)^{1-(\ell+2) / 2^{r}} .
$$

Pour $\ell=R-1$, nous obtenons essentiellement (1.5) mais où le terme principal est multiplié par $H:=H_{0} \cdots H_{R}$. Or, le cas $t_{j}=1(0 \leqslant j<r)$ fournit immédiatement $H=1$, par intégrations successives et utilisation itérée de la formule d'Euler pour la fonction Bêta. 
Remarque. On retrouve bien le terme principal de $S_{r-1}(N ; \boldsymbol{t})$ en choisissant $t_{r-1}=1$ dans celui de $S_{r}(N ; \boldsymbol{t})$. En effet, dans cette circonstance, la seule condition sur la variable $v_{2^{r-1}}$ est

$$
v_{2^{r-1}} \leqslant 1-\sum_{\substack{1 \leqslant h \leqslant R \\ h \neq 2^{r-1}}} v_{h}
$$

Il vient

$$
\begin{aligned}
\frac{1}{\Gamma\left(1 / 2^{r}\right)^{2^{r}}} & \int \frac{\mathrm{d} \boldsymbol{v}}{\prod_{1 \leqslant h \leqslant R} v_{h}^{1-1 / 2^{r}}\left(1-\sum_{1 \leqslant h \leqslant R} v_{h}\right)^{1-1 / 2^{r}}} \\
& =\frac{1}{\Gamma\left(1 / 2^{r}\right)^{2^{r}-2} \Gamma\left(1 / 2^{r-1}\right)} \int \frac{\mathrm{d} \boldsymbol{v}}{\prod_{\substack{1 \leqslant h \leqslant R \\
h \neq 2^{r-1}}} v_{h}^{1-1 / 2^{r}}\left(1-\sum_{\substack{1 \leqslant h \leqslant R \\
h \neq 2^{r-1}}} v_{h}\right)^{1-1 / 2^{r-1}}} .
\end{aligned}
$$

Pour chaque indice $h \leqslant 2^{r-1}-1$, nous effectuons alors le changement de variables $w_{h}=v_{h}+v_{2^{r-1}+h}$ et observons que

$$
\int_{0}^{w_{h}} \frac{\mathrm{d} v_{2^{r-1}+h}}{\left\{v_{2^{r-1}+h}\left(w_{h}-v_{2^{r-1}+h}\right)\right\}^{1-1 / 2^{r}}}=\frac{\Gamma\left(1 / 2^{r}\right)^{2}}{\Gamma\left(1 / 2^{r-1}\right) w_{h}^{1-1 / 2^{r-1}}} .
$$

Par insertion dans l'intégrale relative à $h \leqslant 2^{r-1}-1$, nous obtenons l'expression

$$
\frac{1}{\Gamma\left(1 / 2^{r-1}\right)^{2^{r-1}}} \int \frac{\mathrm{d} \boldsymbol{v}}{\prod_{1 \leqslant h \leqslant 2^{r-1}-1} w_{h}^{1-1 / 2^{r-1}}\left(1-\sum_{1 \leqslant h \leqslant 2^{r-1}-1} w_{h}\right)^{1-1 / 2^{r-1}}}
$$

où le domaine d'intégration est bien $D\left(t_{0}, \cdots, t_{r-2}\right)$.

\section{Démonstration du Théorème 1.2}

Pour chaque couple d'entiers positifs $(m, d)$, posons

$$
\lambda_{m, d}(k):=\frac{\tau(m) \tau(m d)}{\tau(k m) \tau(k m d)} \quad(k \geqslant 1) .
$$

Alors $\lambda_{m, d}$ est multiplicative et nous avons

$$
\begin{aligned}
\sum_{k \geqslant 1} \frac{\lambda_{m, d}(k)}{k^{s}} & =\zeta(s)^{1 / 4} H_{m, d}(s), \\
H_{m, d}(s) & :=\prod_{p}\left(1-\frac{1}{p^{s}}\right)^{1 / 4} \sum_{\nu \geqslant 0} \frac{\left\{v_{p}(m)+1\right\}\left\{v_{p}(m d)+1\right\}}{\left\{v_{p}(m)+\nu+1\right\}\left\{v_{p}(m d)+\nu+1\right\} p^{\nu s}},
\end{aligned}
$$

où $v_{p}$ désigne la valuation $p$-adique. Posant

$$
S^{*}(N ; u, v):=\frac{1}{N} \sum_{d \leqslant N^{u}, m \leqslant N^{v}} \frac{1}{\tau(m) \tau(m d)} \sum_{k \leqslant N / m d} \lambda_{m, d}(k),
$$

nous avons

$$
S(N ; u, v)=S^{*}(N ; u, v)+O\left(1 /(\log N)^{1 / 4}\right),
$$

où l'erreur provient du remplacement, dans la définition (1.8), des bornes $n^{u}$ et $n^{v}$ par $N^{u}$ et $N^{v}$

Considérons d'abord le cas $u+v \leqslant 1$, pour lequel nous avons $\left.D_{u, v}=\right] 0, u[\times] 0, v[$. Sans restreindre la généralité, nous pouvons alors supposer que l'on a

$$
\min (u, v, 1-u-v) \geqslant(\log 2) / \log N \text {. }
$$

En effet, $\sin \min (u, v)<(\log 2) / \log N$ nous avons $\min (d, m)=1$ dans la sommation de $(3 \cdot 1)$ et l'on constate aisément que $S^{*}(N ; u, v)$ et le terme principal de (1.9) sont englobés par le terme d'erreur. Lorsque $v>w:=1-u-(\log 2) / \log N$, on note que seul $k=1$ apparaît dans la somme intérieure de $S^{*}(N ; u, v)-S^{*}(N ; u, w)$ : l'erreur induite est donc facilement majorée. 
Posons $H:=H_{1,1}(1)$, et $g(m, d):=H_{m, d}(1) / H$. Pour tout $\left.\delta \in\right] 0, \frac{1}{2}$ [ et uniformément pour $\Re e s \geqslant 1-\delta$, nous avons $H_{m, d}(s) \ll \varphi_{\delta}(m d)$. Le théorème II.5.2 de [23] implique donc

$$
\sum_{k \leqslant y} \lambda_{m, d}(k)=\frac{H g(m, d)}{\Gamma\left(\frac{1}{4}\right)} \int_{1}^{y} \frac{\mathrm{d} t}{(1+\log t)^{3 / 4}}+O\left(\frac{\varphi_{\delta}(m d) y}{(1+\log y)^{7 / 4}}\right) \quad(y \geqslant 1) .
$$

Insérons cette estimation dans (3·1). La contribution du terme d'erreur est

$$
\begin{aligned}
& \ll \frac{1}{N} \sum_{m d \leqslant N} \frac{\varphi_{\delta}(m d)}{\tau(m) \tau(m d)} \int_{1}^{N / m d} \frac{\mathrm{d} t}{(1+\log t)^{7 / 4}} \\
& =\frac{1}{N} \int_{1}^{N} \sum_{m d \leqslant N / t} \frac{\varphi_{\delta}(m d)}{\tau(m) \tau(m d)} \frac{\mathrm{d} t}{(1+\log t)^{7 / 4}} \\
& \ll \int_{1}^{N} \frac{\mathrm{d} t}{t(1+\log N / t)^{1 / 4}(1+\log t)^{7 / 4}} \ll \frac{1}{(\log N)^{1 / 4}} .
\end{aligned}
$$

Pour évaluer la contribution du terme principal de $(3 \cdot 3)$ au membre de droite de $(3 \cdot 1)$, nous introduisons les fonctions multiplicatives

$$
g(m):=\frac{g(m, 1)}{\tau(m)^{2}}, \quad g_{m}(d):=\frac{g(m, d) \tau(m)}{g(m, 1) \tau(m d)} .
$$

Pour tout $\delta \in] 0, \frac{1}{2}$ [ fixé et uniformément pour $y \geqslant 1$, nous avons

$$
\sum_{d \leqslant y} g_{m}(d)=\frac{G \vartheta(m)}{\Gamma\left(\frac{1}{2}\right)} \int_{1}^{y} \frac{\mathrm{d} s}{\sqrt{1+\log s}}+O\left(\frac{y \varphi_{\delta}(m)}{(1+\log y)^{3 / 2}}\right)
$$

où l'on a posé

$$
G:=\prod_{p}\left(1-\frac{1}{p}\right)^{1 / 2} \sum_{\nu \geqslant 0} \frac{g_{1}\left(p^{\nu}\right)}{p^{\nu}}, \quad \vartheta(m):=\prod_{p^{\mu} \| m} \frac{\sum_{\nu \geqslant 0} g_{p^{\mu}}\left(p^{\nu}\right) / p^{\nu}}{\sum_{\nu \geqslant 0} g_{1}\left(p^{\nu}\right) / p^{\nu}},
$$

et

$$
\sum_{d \leqslant y} g(m) \vartheta(m)=\frac{K y}{\Gamma\left(\frac{1}{4}\right)(1+\log y)^{3 / 4}}+O\left(\frac{y}{(1+\log y)^{7 / 4}}\right)
$$

avec

$$
K:=\prod_{p}\left(1-\frac{1}{p}\right)^{1 / 4} \sum_{\nu \geqslant 0} \frac{g\left(p^{\nu}\right) \vartheta\left(p^{\nu}\right)}{p^{\nu}} .
$$

À fins de référence ultérieure, nous observons que

$$
H G K=\lim _{s \rightarrow 1+} \frac{1}{\zeta(s)} \sum_{\min (m, d, k) \geqslant 1} \frac{1}{\tau(m d k) \tau(m k)(m d k)^{s}}=1
$$

puisque l'on a en fait, pour tout $s>1$,

$$
\sum_{m \geqslant 1, d \geqslant 1, k \geqslant 1} \frac{1}{\tau(m d k) \tau(m k)(m d k)^{s}}=\sum_{n \geqslant 1} \frac{1}{\tau(n) n^{s}} \sum_{m k \mid n} \frac{1}{\tau(m k)}=\zeta(s) .
$$


La contribution du terme d'erreur n'excède pas l'ordre de grandeur obtenu précédemment. Posant $E_{N}:=1 /(\log N)^{1 / 4}$, nous obtenons, compte tenu de $(3 \cdot 4)$,

$$
\begin{aligned}
S(N ; u, v) & =\frac{H}{N \Gamma\left(\frac{1}{4}\right)} \sum_{m \leqslant N^{v}} g(m) \sum_{d \leqslant N^{u}} g_{m}(d) \int_{1}^{N / m d} \frac{\mathrm{d} t}{(1+\log t)^{3 / 4}}+O\left(E_{N}\right) \\
& =\frac{H}{N \Gamma\left(\frac{1}{4}\right)} \sum_{m \leqslant N^{v}} g(m) \int_{1}^{N / m} \sum_{d \leqslant \min \left(N / m t, N^{u}\right)} g_{m}(d) \frac{\mathrm{d} t}{(1+\log t)^{3 / 4}}+O\left(E_{N}\right)
\end{aligned}
$$

La contribution correspondant à la contrainte supplémentaire $m t \leqslant N^{1-u}$ est $\ll E_{N}$. Il suit

$$
\begin{aligned}
& S(N ; u, v)=\frac{H G}{N \Gamma\left(\frac{1}{4}\right) \Gamma\left(\frac{1}{2}\right)} \sum_{m \leqslant N^{v}} \int_{N^{1-u} / m}^{N / m} \frac{g(m) \vartheta(m) \mathrm{d} t}{(1+\log t)^{3 / 4}} \int_{1}^{N / m t} \frac{\mathrm{d} s}{\sqrt{1+\log s}}+O\left(E_{N}\right) \\
& =\frac{H G}{N \Gamma\left(\frac{1}{4}\right) \Gamma\left(\frac{1}{2}\right)} \int_{N^{1-u-v}}^{N} \frac{\mathrm{d} t}{(1+\log t)^{3 / 4}} \int_{1}^{\min \left(N / t, N^{u}\right)} \sum_{\substack{m \leqslant \min \left(N / s t, N^{v}\right) \\
N^{1-u} / t<m}} \frac{g(m) \vartheta(m) \mathrm{d} s}{\sqrt{1+\log s}}+O\left(E_{N}\right) .
\end{aligned}
$$

Dans cette intégrale double, les contributions respectives correspondant aux conditions supplémentaires $s t \leqslant N^{1-v}$ et $t \leqslant N^{1-u}$ peuvent également être englobée dans le terme d'erreur. Nous obtenons

$$
\begin{aligned}
& S(N ; u, v) \\
& =\frac{H G}{N \Gamma\left(\frac{1}{4}\right) \Gamma\left(\frac{1}{2}\right)} \int_{N^{1-u-v}}^{N} \frac{\mathrm{d} t}{(1+\log t)^{3 / 4}} \int_{N^{1-v} / t}^{\min \left(N / t, N^{u}\right)} \sum_{m \leqslant N / s t} \frac{g(m) \vartheta(m) \mathrm{d} s}{\sqrt{1+\log s}}+O\left(E_{N}\right) \\
& =\frac{H G K}{\Gamma\left(\frac{1}{4}\right)^{2} \Gamma\left(\frac{1}{2}\right)} \int_{N^{1-u-v}}^{N} \frac{\mathrm{d} t}{t(1+\log t)^{3 / 4}} \int_{N^{1-v} / t}^{\min \left(N / t, N^{u}\right)} \frac{\mathrm{d} s}{s\{1+\log (N / s t)\}^{3 / 4} \sqrt{1+\log s}}+O\left(E_{N}\right) \\
& =\frac{1}{\Gamma\left(\frac{1}{4}\right)^{2} \Gamma\left(\frac{1}{2}\right)} \int_{1-u-v}^{1} \frac{\mathrm{d} w}{w^{3 / 4}} \int_{\max (0,1-v-w)}^{\min (1-w, u)} \frac{\mathrm{d} z}{(1-w-z)^{3 / 4} z^{1 / 2}}+O\left(E_{N}\right) \\
& =\frac{1}{\Gamma\left(\frac{1}{4}\right)^{2} \Gamma\left(\frac{1}{2}\right)} \int_{0}^{u} \frac{\mathrm{d} s}{\sqrt{s}} \int_{0}^{v} \frac{\mathrm{d} t}{(1-s-t)^{3 / 4} t^{3 / 4}}+O\left(E_{N}\right),
\end{aligned}
$$

où la dernière ligne est obtenue via le changement de variables $(s, t)=(z, 1-w-z)$. Cela fournit bien (1.9) lorsque $u+v \leqslant 1$.

Lorsque $u+v>1$, nous observons que

$$
\begin{aligned}
S^{*}(N ; u, v) & =S^{*}(N ; 1-v, v)+\frac{1}{N} \sum_{\substack{k d m \leqslant N \\
N^{1-v}<d \leqslant N^{u}}} \frac{1}{\tau(k d m) \tau(k m)} \\
& =S^{*}(N ; 1-v, v)+\frac{1}{N} \sum_{\substack{\ell m \leqslant N \\
N^{1-v}<m \leqslant N^{u}}} \frac{1}{\tau(\ell m)} .
\end{aligned}
$$

Compte tenu de ce qui précède, la loi de l'arcsinus fournit alors, pour $u+v>1$,

$$
\begin{aligned}
S(N ; u, v)= & \frac{1}{\Gamma\left(\frac{1}{4}\right)^{2} \Gamma\left(\frac{1}{2}\right)} \int_{0}^{1-v} \frac{\mathrm{d} s}{\sqrt{s}} \int_{0}^{v} \frac{\mathrm{d} t}{(1-s-t)^{3 / 4} t^{3 / 4}} \\
& +\frac{2}{\pi}(\arcsin \sqrt{u}-\arcsin \sqrt{1-v})+O\left(\frac{1}{(\log N)^{1 / 4}}\right) .
\end{aligned}
$$


Cependant, d'après le théorème II.0.8 et le corollaire II.0.9 de [23], nous obtenons, via un changement de variables adéquat,

$$
\begin{aligned}
\frac{1}{\Gamma\left(\frac{1}{4}\right)^{2} \Gamma\left(\frac{1}{2}\right)} & \int_{1-v}^{u} \frac{\mathrm{d} s}{\sqrt{s}} \int_{0}^{1-s} \frac{\mathrm{d} t}{(1-s-t)^{3 / 4} t^{3 / 4}} \\
& =\frac{1}{\Gamma\left(\frac{1}{4}\right)^{2} \Gamma\left(\frac{1}{2}\right)} \int_{1-v}^{u} \frac{\mathrm{d} s}{\sqrt{s(1-s)}} \int_{0}^{1} \frac{\mathrm{d} w}{(1-w)^{3 / 4} w^{3 / 4}} \\
& =\frac{1}{\pi} \int_{1-v}^{u} \frac{\mathrm{d} s}{\sqrt{s(1-s)}}=\frac{2}{\pi}(\arcsin \sqrt{u}-\arcsin \sqrt{1-v}) .
\end{aligned}
$$

Cela achève la démonstration.

\section{Preuve du Corollaire $\mathbf{1 . 3}$}

Compte tenu de (1.9) et de l'expression classique de $B\left(\frac{3}{4}, \frac{1}{2}\right)$ (cf., par exemple, le théorème II.0.8 de [23]), nous obtenons

$$
\begin{aligned}
S(N ; v)=S(N ; 1, v) & =\frac{1}{\Gamma\left(\frac{1}{4}\right)^{2} \Gamma\left(\frac{1}{2}\right)} \int_{0}^{v} \frac{\mathrm{d} t}{t^{3 / 4}} \int_{0}^{1-t} \frac{\mathrm{d} s}{(1-s-t)^{3 / 4} s^{1 / 2}}+O\left(\frac{1}{(\log N)^{1 / 4}}\right) \\
& =\frac{1}{\Gamma\left(\frac{1}{4}\right) \Gamma\left(\frac{3}{4}\right)} \int_{0}^{v} \frac{\mathrm{d} w}{t^{3 / 4}(1-t)^{1 / 4}}+O\left(\frac{1}{(\log N)^{1 / 4}}\right) .
\end{aligned}
$$

Cela implique le résultat annoncé via la formule des compléments.

Remerciements. Les auteurs expriment leur gratitude envers l'arbitre pour de judicieuses suggestions. Le second auteur tient de plus à remercier Eugenijus Manstavičius pour les éclaircissements prodigués relativement à certains éléments de la bibliographie.

\section{Bibliographie}

[1] G.J. Babu, A Note on the Invariance Principle for Additive Functions, The Indian Journal of Statistics, Series A, 35 no 3 (1973), 307-310.

[2] G. Bareikis \& A. Mačiulis, Cesàro means related to the square of the divisor function, Acta Arith. 156 (2012), 83-99.

[3] Bareikis \& A. Mačiulis, On the second moment of an arithmetical process related to the natural divisors, Ramanujan J. 37, 1-24.

[4] G. Bareikis \& E. Manstavičius, On the DDT theorem, Acta Arith. 126 no 2 (2007), 155-168.

[5] P. Billingsley, Additive functions and Brownian motion, Notices Amer. Math. Soc. 17 (1970), 1050.

[6] P. Billingsley, The Probability Theory of Additive Arithmetic Functions, The Annals of Probability 2 n 5 (1974), 749-791.

[7] J.-M. Deshouillers, F. Dress, G. Tenenbaum, Lois de répartition des diviseurs, I, Acta Arith. 34 (1979), 273-285.

[8] P. Erdős \& M. Kac, On the number of positive sums of independent random variables, Bull. Amer. Math. Soc. 53 (1947), 1011-1020.

[9] W.J. Feller, An introduction to probability theory and its applications, vol. 1, John Wiley, 1970 (1ère éd. 1950).

[10] T.S. Ferguson, A Bayesian analysis of some nonparametric problems, Ann. Stat. 1 no 2 (1973), 209-230.

[11] R.R. Hall, Sets of multiples, Cambridge Tracts in Mathematics 118, Cambridge University Press, Cambridge, 1996, xvi+264 pp.

[12] R.R. Hall \& G. Tenenbaum, Divisors, Cambridge Tracts in Mathematics 90, Cambridge 1988.

[13] U.M. Hirth, Probabilistic number theory, the GEM/Poisson-Dirichlet distribution and the arcsine law, Combin. Probab. Comput. 6 no 1 (1997), 57-77.

[14] J. Kubilius, Méthodes probabilistes en théorie des nombres (en russe), Uspehi Mat. Nauk 11, no 2 (1956), 31-66 = Amer. Math. Soc. Transl. 19 (1962), 47-85. 
[15] J. Kubilius, Probabilistic methods in the theory of numbers, Amer. Math. Soc. Translations of Math. Monographs, $n^{\circ} 11$ (1964), Providence.

[16] E. Manstavičius, An invariance principle for additive arithmetic functions (en russe), Dokl. Akad. Nauk SSSR 298 (1988), no 6, 1316-1320; traduction anglaise : Soviet Math. Dokl. 37 (1988), $\mathrm{n}^{\mathrm{o}} 1,259-263$.

[17] E. Manstavičius, Natural divisors and the Brownian motion, J. Théor. Nombres Bordeaux $8 \mathrm{n}^{\circ} 1$ (1996), 159-171.

[18] E. Manstavičius \& N.M. Timofeev, A functional limit theorem related to natural divisors. Acta Math. Hungar. 75 (1997), $\mathrm{n}^{\text {os }} 1-2,1-13$.

[19] S. Nyandwi \& A. Smati, Distribution laws of pairs of divisors, Integers 13 (2013), A13.

[20] W. Philipp, Arithmetic functions and Brownian motion, in : Analytic Number Theory, Proc. Sympos. Pure Math. (St. Louis 1972) 24 (1973), 233-246.

[21] G. Tenenbaum, Lois de répartition des diviseurs, 4, Ann. Inst. Fourier 29, 3 (1979), 1-15.

[22] G. Tenenbaum, Addendum to the paper of E. Manstavičius \& M.N. Timofeev "Functional limit theorem related to natural divisors", Acta Math. Hungar. 75, nos. 1-2 (1997), 15-22.

[23] G. Tenenbaum, Introduction à la théorie analytique et probabiliste des nombres, quatrième édition, coll. Échelles, Belin, 2015. (English translation : Graduate Studies in Mathematics 163, Amer. Math. Soc. 2015 ; 2nd ed., English, Cambridge Studies Adv. Math. 46, Cambridge Univ. Press, 1995).

[24] N.M. Timofeev \& Kh.Kh. Usmanov, Arithmetic modeling of Brownian motion (en russe) Dokl. Akad. Nauk Tadzhik. SSR 25 n 4 (1982), 207-211.

Régis de la Bretèche

Université Paris Diderot-Paris 7

Sorbonne Paris Cité, UMR 7586

Institut de Mathématiques de Jussieu-PRG

Case 7012, F-75013 Paris

regis.delabreteche@imj-prg.fr

\author{
Gérald Tenenbaum \\ Institut élie Cartan \\ Université de Lorraine \\ BP 70239 \\ F-54506 Vandœuvre-lès-Nancy Cedex \\ gerald.tenenbaum@univ-lorraine.fr
}

